\title{
Editorial
}

\section{Recent Advances in High Throughput Sequencing Analysis}

\author{
Yan Guo, ${ }^{1}$ Leng Han, ${ }^{2}$ and Quanhu Sheng ${ }^{1}$ \\ ${ }^{1}$ Department of Biomedical Informatics, Vanderbilt University Medical Center, Nashville, TN 37027, USA \\ ${ }^{2}$ Department of Biochemistry and Molecular Biology, University of Texas Medical School, Houston, TX 77030, USA
}

Correspondence should be addressed to Yan Guo; yan.guo@vanderbilt.edu

Received 22 March 2017; Accepted 22 March 2017; Published 19 June 2017

Copyright $\odot 2017$ Yan Guo et al. This is an open access article distributed under the Creative Commons Attribution License, which permits unrestricted use, distribution, and reproduction in any medium, provided the original work is properly cited.

High-throughput genomic technology has enabled us to screen the entire genome and generate hypotheses at relatively low costs. One of the driving forces in highthroughput genomic technology is high-throughput sequencing (HTS). With its rapid development and affordability, HTS has quickly become the go-to choice for interrogating the entire genome. The analysis methodology development for HTS has been at the forefront of bioinformatics in recent years. Hundreds of tools and pipelines have been developed to aid researchers in interpreting HTS data. The aim of this special issue is to promote research and reflect the most recent advances in addressing HTS data analysis.

We received a total of 14 manuscripts and, through rigorous review, selected six for publication in this special issue. What follows is a brief summary of the six manuscripts:

(1) Title: "Chromosome 1 Sequence Analysis of C57BL/ 6J-Chr $1{ }^{\mathrm{KM}}$ Mouse Strain." In this article, the authors studied the chromosome 1 sequence of the Chinese Kunming mouse and compared the sequence to three other mouse species.

(2) Title: "The Utilization of Formalin Fixed-ParaffinEmbedded Specimens in High Throughput Genomic Studies." In this review article, the authors thoroughly examined the practicability of conducting highthroughput genomic assays, including HTS using formalin-fixed paraffin-embedded specimens.

(3) Title: "An Integrating Approach for Genome-Wide Screening of MicroRNA Polymorphisms Mediated Drug Response Alterations." In this study, the authors examined the relationship between polymorphisms and drug response in microRNA.

(4) Title: "Comparative Transcriptome Analysis Reveals Effects of Exogenous Hematin on Anthocyanin Biosynthesis during Strawberry Fruit Ripening." Through RNA sequencing, the authors examined the expression change of genes in strawberries that had been applied with exogenous hematin.

(5) Title: "Differential Gene Expression during Larval Metamorphic Development in the Pearl Oyster, Pinctada fucata, Based on Transcriptome Analysis." Through RNA sequencing, the authors studied changes in the gene expression pattern during the metamorphic development of a pearl oyster.

(6) Title: "RNA Sequencing of Formalin-Fixed, ParaffinEmbedded Specimens for Gene Expression Quantification and Data Mining." In this study, the authors examined the efficiency of two ribosomal RNA deletion kits: Ribo-Zero and RNase $\mathrm{H}$.

\section{Acknowledgments}

We would like to extend our gratitude to all authors who contributed to this special issue and all reviewers that helped us select the highest quality manuscripts.

Yan Guo

Leng Han

Quanhu Sheng 

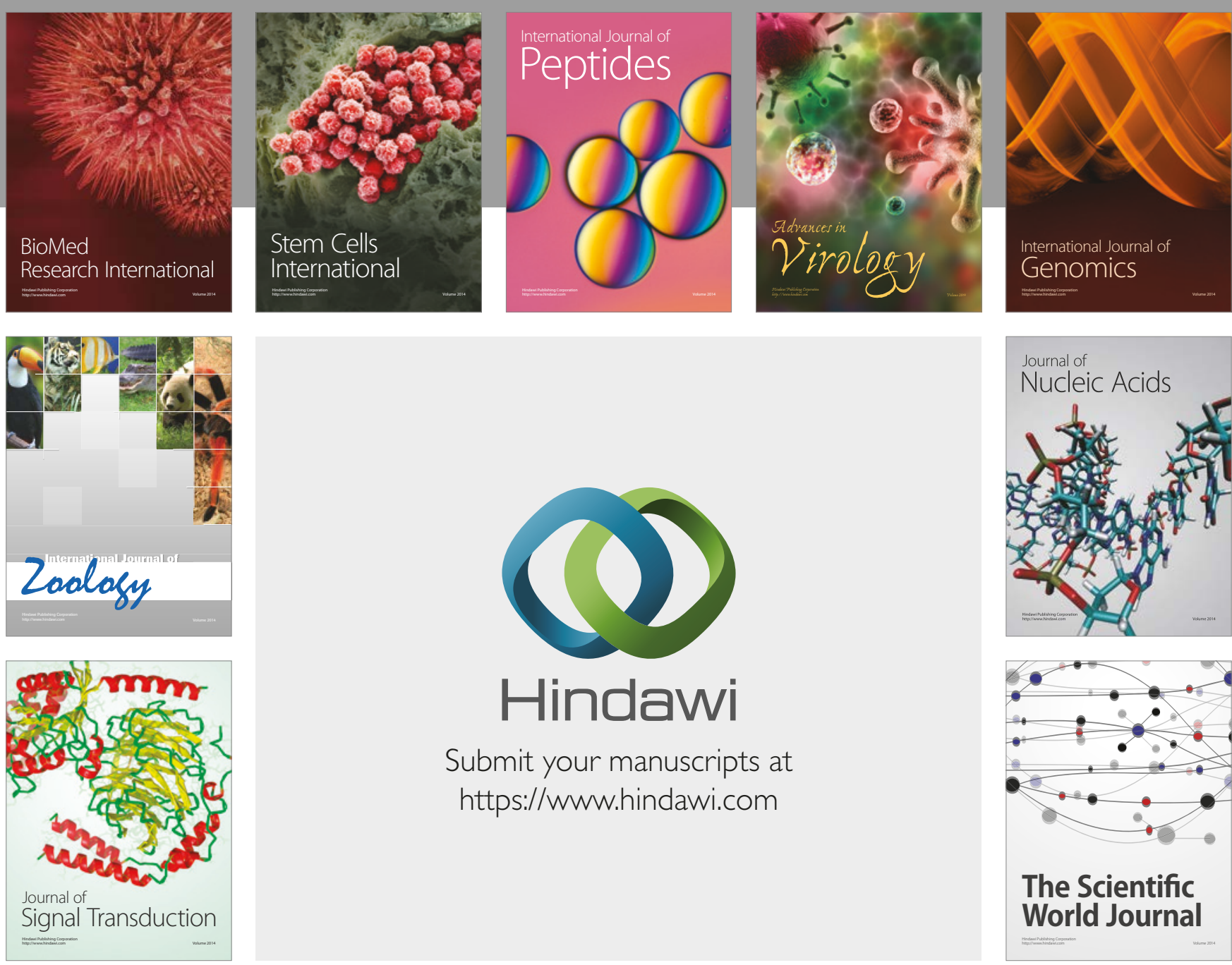

Submit your manuscripts at

https://www.hindawi.com
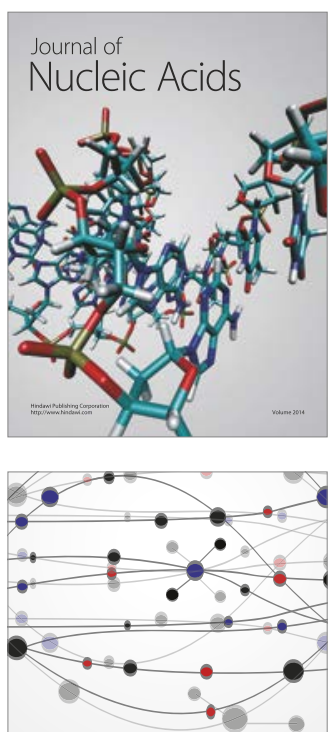

The Scientific World Journal

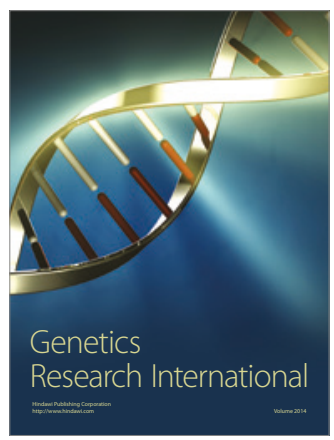

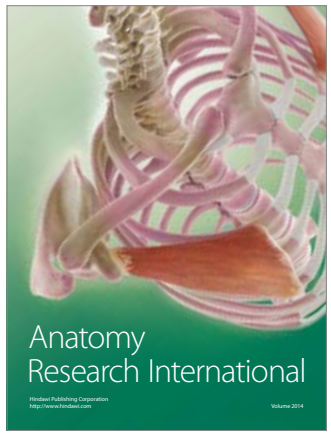

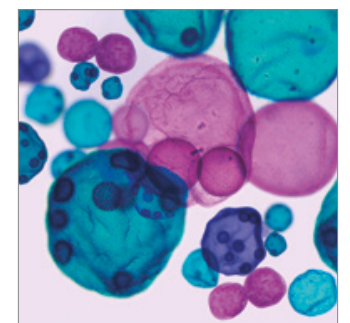

International Journal of Microbiology
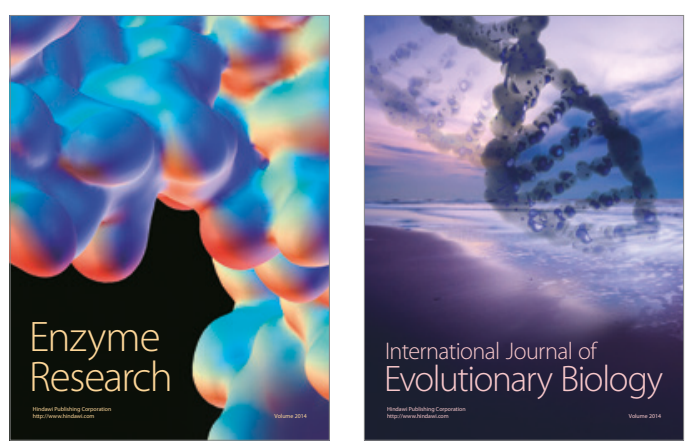
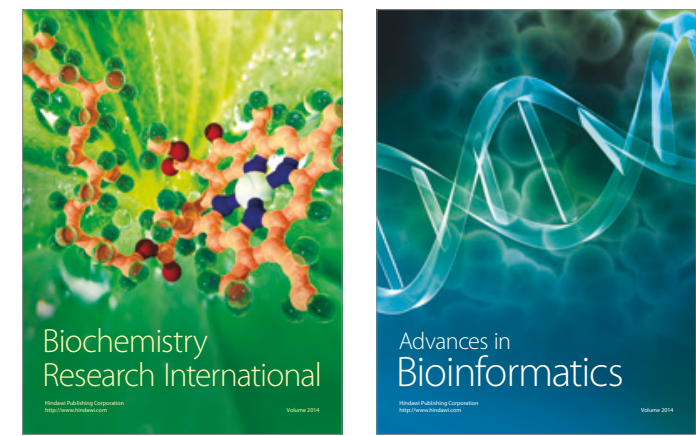

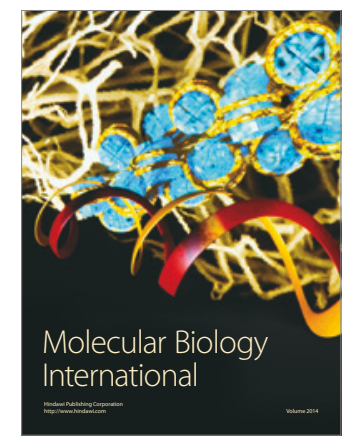

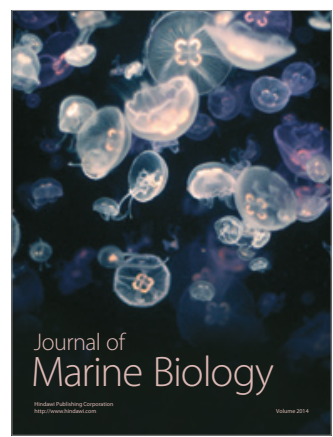

